Marquette University

e-Publications@Marquette

Chemistry Faculty Research and Publications

Chemistry, Department of

$5-1-2006$

\title{
Faithful Estimation of Dynamics Parameters from CPMG Relaxation Dispersion Measurements
}

Evgueni Kovriguine

Marquette University, evgueni.kovriguine@marquette.edu

James G. Kempf

Yale University

Michael J. Grey

Columbia University

J. Patrick Loria

Yale University

Accepted version. Journal of Magnetic Resonance, Vol. 180, No. 1 (May 2006): 93-104. DOI: (C) 2006

Elsevier Inc. Used with permission. 


\section{Marquette University}

\section{e-Publications@Marquette}

\section{Chemistry Faculty Research and Publications/College of Arts and Sciences}

This paper is NOT THE PUBLISHED VERSION; but the author's final, peer-reviewed manuscript. The published version may be accessed by following the link in the citation below.

Journal of Magnetic Resonance, Vol. 180, No. 1 (May 2006): 93-104. DOI. This article is (C) Elsevier and permission has been granted for this version to appear in e-Publications@Marquette. Elsevier does not grant permission for this article to be further copied/distributed or hosted elsewhere without the express permission from Elsevier.

\section{Faithful estimation of dynamics parameters from CPMG relaxation dispersion measurements}

Evgenii L. Kovrigin

Department of Chemistry, Marquette University, Milwaukee, WI

Department of Chemistry, Yale University, P.O. Box 208107, New Haven, CT

James G. Kempf

Department of Chemistry, Yale University, P.O. Box 208107, New Haven, CT

Michael J. Grey

Department of Biochemistry and Molecular Biophysics, Columbia University, 630 W. 168th St., New York, NY

J. Patrick Loria

Department of Chemistry, Yale University, P.O. Box 208107, New Haven, CT

\section{Abstract}

This work examines the robustness of fitting of parameters describing conformational exchange $\left(k_{\mathrm{ex}}, p_{a / b}\right.$, and $\Delta \omega)$ processes from CPMG relaxation dispersion data. We have analyzed the equations describing conformational exchange processes for the intrinsic inter-dependence of their parameters that leads to the 
existence of multiple equivalent solutions, which equally satisfy the experimental data. We have used MonteCarlo simulations and fitting to the synthetic data sets as well as the direct 3-D mapping of the parameter space of $k_{\text {ex }}, p_{a / b}$, and $\Delta \omega$ to quantitatively assess the degree of the parameter inter-dependence. The demonstrated high correlation between parameters can preclude accurate dynamics parameter estimation from NMR spinrelaxation data obtained at a single static magnetic field. The strong parameter inter-dependence can readily be overcome through acquisition of spin-relaxation data at more than one static magnetic field thereby allowing accurate assessment of conformational exchange properties.

\section{Keywords}

Data analysis, CPMG, Protein dynamics, NMR spin-relaxation

\section{Introduction}

The role that molecular motion plays in a multitude of biological processes is well appreciated, while the complexity of such motions can render their characterization quite elusive and uncertain in accuracy. In recent years, it has been demonstrated that solution nuclear magnetic resonance (NMR) experiments have the unique ability to quantitate these motions over a wide range of timescales covering such important biological processes as enzyme catalysis [1], [2], [3], [4], [5], [6], [7], protein folding [8], [9], [10], [11], and binding and recognition [12] [13]. One category of the noted NMR experiments quantitates the dynamics of intramolecular motions or chemical events on the $\mu s-m s$ timescale, which are referred to as conformational or chemical exchange. Exchange events can alter the transverse nuclear-spin-relaxation rate at sites that exhibit a change in chemical shift $(\Delta \omega)$ with the dynamic event. A variety of CPMG-based NMR experiments have been designed for thorough characterization of exchange in proteins. Primarily, these measure the decay of single, zero, and multiple quantum spin-coherences (reviewed in [14], [15]), which are then interpreted in terms of motional parameters. These experiments are in increasingly common usage in attempts to establish a relation between dynamic motions and biological function. Therefore, the accuracy of dynamics' parameter estimation from NMR relaxation studies is crucial for drawing reliable conclusions on the role of dynamics in function.

Estimation of exchange parameters by these NMR methods relies on the variation in the measured transverse relaxation rate constants, $R_{2}$, with $\tau_{\mathrm{cp}}$, the spacing between the $180^{\circ}$ pulses in a CPMG spin-echo sequence [16], [17], [18]. This approach is commonly called relaxation dispersion analysis. Early on, Allerhand et al. [19] recognized the importance of experimental details for obtaining accurate results. More recent reviews offer guidance for avoiding spurious results and artifacts in dispersion methods [14]. However, potential pitfalls remain in choosing the number and type of experiments required for faithful estimation of dynamics parameters. This issue has not been thoroughly discussed in previous literature and, yet, is particularly concerning in that poor choice of experiments can provide apparently accurate, but incorrect results.

Here, we provide a statistical assessment of the accuracy of dynamics parameters estimated from various sets of CPMG-based relaxation dispersion experiments. Our first approach is to generate noise-incorporated simulated $R_{2}\left(\tau_{\mathrm{cp}}\right)$ dispersion data for a given test case of exchange. This result is then compared to dispersion curves generated using dynamics parameters over a broad range of physically reasonable exchange conditions. In the ideal, only those parameter values that closely match the inputs to the original simulation would return dispersion curves sufficiently similar to the noise-incorporated synthetic data. Secondly, direct mapping of the exchange parameters using a grid search over a broader range of parameter spaces tests, in fact, whether any dissimilar conditions reproduce the synthetic result and therefore cast doubt on the fidelity of the experimental method considered.

The results of this process demonstrate that relaxation dispersion data obtained at a single static magnetic field is insufficient to describe the dynamic parameters for a two-site $(a \leftrightarrow b)$ slow exchange process. More accurate estimates the dynamics parameters are obtained from data acquired at two static fields. We also extend our 
analysis to the case of three-site exchange in the fast limit, where the motional rate, $k_{\text {ex }}$ is greater than $\Delta \omega$. The cases described here provide a framework for confident, reliable assessment of dynamics parameters from multi-field $R_{2}\left(\tau_{\mathrm{cp}}\right)$ dispersion measurements.

\section{Materials and methods}

\subsection{Theory}

Exchange in the $\mu s-m s$ time regime results in enhanced transverse relaxation rates. An understanding of the enhancement is necessary to draw the connection between $R_{2}\left(\tau_{\mathrm{cp}}\right)$ dispersion and the parameters of dynamic events. The increase in the observed $R_{2}$ occurs when chemical exchange between sites changes the precessional frequency of the nuclear spin (i.e., $\Delta \omega \neq 0$ ) to result in dephasing of the spin coherence. CPMG spin-echo pulse trains can mitigate this coherence loss. If the CPMG pulsing rate is fast relative to the mean lifetime of the exchange event, then the exchange-induced dephasing is negligible and $R_{2} \sim R_{2}^{0}$, where $R_{2}^{0}$ is the exchange-free transverse relaxation rate constant. However, when exchange events are on the timescale of or faster than the repetition rate of $180^{\circ}$ pulses, the related dephasing significantly contributes to coherence loss, which manifests as an elevated $R_{2}=R_{2}^{0}+R_{\mathrm{ex}}$, where $R_{\mathrm{ex}}$ is the contribution from exchange-induced dephasing.

In a two-site exchange mechanism, a nuclear spin exchanges between sites, $a$ and $b$, that differ in chemical shift by $\Delta \omega=\Omega_{b}-\Omega_{b}$, where $\Omega_{i}$ is the chemical shift for the spin in site $i$. The kinetics of the exchange reaction are described by the apparent exchange rate constant $k_{\mathrm{ex}}=k_{1}+k_{-1}$, where $k_{1}$ and $k_{-1}$ are the first-order rate constants for the forward and reverse transitions, respectively. In the limit of fast exchange, $k_{\text {ex }} \gg \Delta \omega$, the dependence of the measured transverse relaxation rate on the time delay between spin-echo refocusing pulses, $\tau_{\mathrm{cp}}$, is given by [18],

$$
R_{2}\left(1 / \tau_{\mathrm{cp}}\right)=R_{2}^{0}+\Phi_{\mathrm{ex}} / k_{\mathrm{ex}}\left[1-2 \tanh \left(k_{\mathrm{ex}} \tau_{\mathrm{cp}} / 2\right) /\left(k_{\mathrm{ex}} \tau_{\mathrm{cp}}\right)\right] .
$$

In Eq. (1), $\Phi_{\text {ex }}=\Delta \omega^{2} p_{a} p_{b}$ in which $p_{i}$ is the fractional population of the spin in site $i$ and $p_{a}+p_{b}=1$. Eq. (1) is commonly referred to the fast-limit equation because it was derived with this condition in mind. In practice, Eq. (1) is valid for some conditions outside this restriction [20]. Alternatively, a completely general, albeit more complex expression for $R_{2}\left(\tau_{\mathrm{cp}}\right)$ is given by [21], [22], [23],

$$
R_{2}\left(\tau_{\mathrm{cp}}\right)=R_{2}^{0}+\frac{1}{2}\left(k_{\mathrm{ex}}-\frac{1}{\tau_{\mathrm{cp}}} \cosh ^{-1}\left[D_{+} \cosh \left(\eta_{+}\right)-D_{-} \cos \left(\eta_{-}\right)\right]\right),
$$

where

$$
\begin{gathered}
D_{ \pm}=\frac{1}{2}\left[ \pm 1+\frac{\Psi+2 \Delta \omega^{2}}{\left(\Psi^{2}+\zeta^{2}\right)^{1 / 2}}\right] \\
\eta_{ \pm}=\frac{\tau_{\mathrm{cp}}}{\sqrt{2}}\left[ \pm \Psi+\left(\Psi^{2}+\zeta^{2}\right)^{1 / 2}\right]^{1 / 2} \\
\Psi=\left(p_{a} k_{\mathrm{ex}}+p_{b} k_{\mathrm{ex}}\right)^{2}-\Delta \omega^{2}+4 p_{a} p_{b} k_{\mathrm{ex}}^{2}
\end{gathered}
$$

and

$$
\zeta=2 \Delta \omega\left(p_{a} k_{\mathrm{ex}}+p_{b} k_{\mathrm{ex}}\right)
$$


The parameters in Eqs. (2), (3), (4), (5), (6) are as defined for Eq. (1).

Although two-site exchange mechanisms are, by far, the most commonly considered in the literature, recent studies demonstrated the existence of three-site exchange in biological systems [8], [10], [24] For practical reasons, this paper will address three-site exchange for the case of fast-limit, linear three-site processes only. These are defined by

$$
c \underset{k_{2}}{\stackrel{k_{-2}}{\rightleftarrows}} a \underset{k_{-1}}{\stackrel{k_{1}}{\rightleftarrows}} b
$$

Here, the exchange contribution to the measured transverse relaxation rate is [24]

$$
\begin{aligned}
R_{\mathrm{ex}}\left(\tau_{\mathrm{cp}}\right)= & \phi_{2} / \kappa_{2}\left[1-2 \tanh \left(\kappa_{2} \tau_{\mathrm{cp}} / 2\right) /\left(\kappa_{2} \tau_{\mathrm{cp}}\right)\right] \\
& +\phi_{3} / \kappa_{3}\left[1-2 \tanh \left(\kappa_{3} \tau_{\mathrm{cp}} / 2\right) /\left(\kappa_{3} \tau_{\mathrm{cp}}\right)\right]
\end{aligned}
$$

where

$$
\left\{\kappa_{2}, \kappa_{3}\right\}=\left\{\left(k_{\mathrm{ex}}+Z\right),\left(k_{\mathrm{ex}}-Z\right)\right\} / 2
$$

are the apparent exchange rate constants for $a \leftrightarrow c$ and $a \leftrightarrow b$, respectively, and the remaining factors are given by

$$
\begin{gathered}
k_{\mathrm{ex}}=k_{1}+k_{-1}+k_{2}+k_{-2}, \\
Z=\left(k_{\mathrm{ex}}^{2}-4 B\right)^{1 / 2}, \\
B=k_{-1} k_{-2}+k_{1} k_{-2}+k_{-1} k_{2}, \\
\left\{\phi_{2}, \phi_{3}\right\}=\left\{\left(-\kappa_{3} \alpha_{1}+\alpha_{2}\right),\left(\kappa_{2} \alpha_{1}+\alpha_{2}\right)\right\} / Z, \\
\alpha_{1}=p_{a} p_{b}\left(\Omega_{b}-\Omega_{a}\right)^{2}+p_{b} p_{c}\left(\Omega_{c}-\Omega_{b}\right)^{2}+p_{a} p_{c}\left(\Omega_{c}-\Omega_{a}\right)^{2}, \\
\alpha_{2}=p_{a}\left[k_{1}\left(\Omega_{b}-\Omega_{a}\right)^{2}+k_{2}\left(\Omega_{c}-\Omega_{a}\right)^{2}\right],
\end{gathered}
$$

and

$$
p_{a}=k_{-1} k_{-2} / B ; p_{b}=k_{1} k_{-2} / B ; p_{c}=k_{-1} k_{2} / B
$$

In the limit where the kinetic timescales for the two exchange processes differ by at least an order of magnitude, the exchange contributions for each process can be treated independently and $\mathrm{k} 2 \approx \mathrm{k} 2+\mathrm{k}-2$ and $\mathrm{k} 3 \approx \mathrm{k} 1+\mathrm{k}-1$. Outside the fast limit, a numerical solution to the three-site exchange problem is necessary [8], [24].

\subsection{Exchange scenarios}

We considered eight different cases of exchange in two major categories: two-site and three-site exchange. For two-site processes, we treated a single amino-acid residue undergoing either (1) slow $\left(k_{\text {ex }}<\Delta \omega\right),(2)$ intermediate $\left(k_{\mathrm{ex}} \sim \Delta \omega\right)$, or (3) fast $\left(k_{\mathrm{ex}}>\Delta \omega\right)$ exchange. In these three cases, it was assumed that dispersion data were collected at only a single value of the static magnetic field. An additional single-field scenario was treated 
in which (4) three separate amino-acid residues undergo a global exchange process, meaning they share common values of $k_{\text {ex }}$ and $p_{a}$, while $\Delta \omega$ and $R_{2}^{0}$ are allowed residue-specific values.

The benefits of collecting $R_{2}\left(\tau_{\mathrm{cp}}\right)$ dispersion data at multiple static magnetic fields are in the increased size of the data set to be fit and, most importantly, in the reduction of parameter correlations due to the distinct fielddependencies of parameters. We assessed the extent of this benefit for test cases in which $R_{2}\left(\tau_{\mathrm{cp}}\right)$ dispersion data is available from (5) two and (6) three static magnetic fields, both for single-residue exchange. Here, $\Delta \omega$ and $R_{2}^{0}$ are constrained by their field dependence, e.g., $\Delta \omega$ at a field with $800 \mathrm{MHz}{ }^{1} \mathrm{H}$ frequency was restricted to $(800 / 500 \sim 1.6)$ times the value at $500 \mathrm{MHz}$.

In our second major category, three-site linear exchange, we treat a single residue in fast exchange and consider the quality of results obtained assuming data is available from (7) two and (8) three field values. Here, the exchange was defined by parameters chosen for their similarity to those obtained in a recent characterization of three-site exchange [24]. Expressing each in units of $\mathrm{s}^{-1}$, these are: $k_{1}=10, k_{2}=100, k_{-1}=1000$, and $k_{-2}=10,000$, which yields $k_{\mathrm{ex}} \sim 11,000$, and $\left(\Omega_{b}-\Omega_{a}\right)=250,\left(\Omega_{c}-\Omega_{b}\right)=500$, and $\left(\Omega_{c}-\Omega_{a}\right)=750$. These values correspond to $\kappa_{2}=10,100, \kappa_{3}=1010, \phi_{2}=2425$, and $\phi_{3}=203$, and were used to simulate dispersion curves at fields corresponding to 500,600 , and $800 \mathrm{MHz}^{1} \mathrm{H}$ frequency using Eq. (8).

\subsection{Generation of synthetic data}

For each of the eight test cases just presented, we generated 1000 synthetic $R_{2}\left(\tau_{\mathrm{cp}}\right)$ data sets using common exchange parameters and experimental conditions and modified each set by incorporating random errors in the $R_{2}\left(\tau_{\mathrm{cp}}\right)$ values. Error values were selected from a Gaussian noise distribution with full width at half max at $5 \%$ of a given $R_{2}\left(\tau_{\mathrm{cp}}\right)$. Each of the 1000 case-specific sets included 12 error-incorporated $R_{2}\left(\tau_{\mathrm{cp}}\right)$ values, where $\tau_{\mathrm{cp}}$ ranged between $714 \mu \mathrm{s}$ and $65.0 \mathrm{~ms}\left(v_{\mathrm{cpmg}}=\left(1 / \tau_{\mathrm{cp}}\right) \sim\left[1400-15 \mathrm{~s}^{-1}\right]\right)$ to reflect experimentally practical values for ${ }^{15} \mathrm{~N}$ relaxation experiments on state-of-the-art NMR spectrometers.

\subsection{Statistical analyses of synthetic results}

\subsubsection{Nonlinear least-squares fitting}

To assess the quality of exchange parameters that can be expected from experimental data, we fit Eq. (2), the two-site full-exchange expression and Eq. (8), the three-site fast-exchange expression to each of the 1000 casespecific synthetic $R_{2}\left(\tau_{\mathrm{cp}}\right)$. Histogramatic display of the fit results reveals the parameter vales that may appear to characterize an exchange event, regardless of the true dynamics of that event. Nonlinear least-squares fits were executed using Prism 4.0 (GraphPad Software) and were constrained so that (1) $0.8 \leqslant p_{a} \leqslant 1.0$ (i.e., $p_{a} \gg p_{b}$ ), (2)

$R_{2}^{0}>10 \mathrm{~s}^{-1}$, (3) $k_{\mathrm{ex}}>100 \mathrm{~s}^{-1}$, and (4) $\Delta \omega>50 \mathrm{~s}^{-1}$ for two-site exchange. The $R_{2}^{0}$ constraint is reasonable for ${ }^{15} \mathrm{~N}$ nuclei in a macromolecule with rotational correlation time $>3 \mathrm{~ns}$, while limitations on $p_{a}, k_{\mathrm{ex}}$, and $\Delta \omega$ avoid parameter values that do not yield practically observable dispersion or that represent less-common physical conditions (e.g., $p_{a}<0.8$ ). Similar constraints were placed on analogous parameters in the three-site exchange process.

\subsubsection{Direct, comparative mapping of parameter space}

In separate tests, we explored the uniqueness with which a given set of exchange parameters defines $R_{2}\left(\tau_{\mathrm{cp}}\right)$ dispersion by comparing the theoretical $R_{2}\left(\tau_{\mathrm{cp}}\right)$ values that correspond to a given case of exchange with those calculated at points on a multidimensional grid encompassing a broad, physically reasonable parameter space. For cases of two-site exchange analyzed with this approach, the three-dimensional parameter space $\left(k_{\mathrm{ex}}, p_{a}\right.$, and $\Delta \omega$ ) was explored according to the dependence $R_{2}\left(\tau_{\mathrm{cp}}\right)$ in Eq. (2).

Parameter grid locations were considered indistinguishable from the true input values when they reproduced the $R_{2}\left(\tau_{\mathrm{cp}}\right)$ data to within a specified tolerance, which was defined as follows. First, $5 \%$ Gaussian noise was added 
to the synthetic $R_{2}\left(\tau_{\mathrm{cp}}\right)$ data set at 12 values of $\tau_{\mathrm{cp}}$, as described earlier. The rmsd of this error-incorporated set $\left[R_{2}^{5 \%}\left(\tau_{\mathrm{cp}}\right)\right]$ from the unmodified test data $\left[R_{2}^{\text {test }}\left(\tau_{\mathrm{cp}}\right)\right]$ was then calculated as

$$
\Delta_{5 \%}=\sqrt{\sum_{\tau_{\mathrm{cp}}}\left[R_{2}^{\mathrm{test}}\left(\tau_{\mathrm{cp}}\right)-R_{2}^{5 \%}\left(\tau_{\mathrm{cp}}\right)\right]^{2}},
$$

The procedure was repeated 50 times to obtain an average $\Delta_{5 \%}$ value, which is the mean distance of errorincorporated $R_{2}\left(\tau_{\mathrm{cp}}\right)$ sets from the theoretical curve. This distance represents a region about the theoretical curve within which any similar curve cannot be distinguished from the original. Thus, our second step was to calculate,

$$
\Delta_{\text {grid }}=\sqrt{\sum_{\tau_{\mathrm{cp}}}\left[R_{2}^{\text {test }}\left(\tau_{\mathrm{cp}}\right)-R_{2}^{\text {grid }}\left(\tau_{\mathrm{cp}}\right)\right]^{2}}
$$

the rmsd of the $R_{2}\left(\tau_{\mathrm{cp}}\right)$ set at a particular grid point from the original theoretical test curve. Parameter sets (i.e., grid points) that produced $\Delta_{\text {grid }}<\Delta_{5 \%}$ were considered to be indistinguishable from those in the test set.

Finally, to facilitate comparison between both methods, the test cases evaluated using direct mapping are identical to those treated using the nonlinear least-squares fitting of error-incorporated data sets as in (2.2). The parameter space explored for two-site exchange encompassed $k_{\mathrm{ex}}=50-3000 \mathrm{~s}^{-1}, p_{a}=0.75-1$, and $\Delta \omega=100-$ $3000 \mathrm{~s}^{-1}$ in grid increments of $30 \mathrm{~s}^{-1}, 0.0025$, and $30 \mathrm{~s}^{-1}$, respectively. This resulted in an approximately $100 \times 100 \times 100$ data array. The grid search and comparison algorithm was programmed in MatLab 7 and performed on a $3 \mathrm{GHz}$ Pentium-IV computer. This resulted in about 3-5 min computation time for a single test parameter set. The MatLab notebooks are available from the authors upon request.

\section{Results and discussion}

When employing solution-state NMR to study protein dynamics, an intuitive feel for the relation between dynamics parameters and the observed dispersion curves can be an excellent guide in choosing the best experiments for accurate results. Unfortunately, the complexity of Eqs. (1), (2), (8) conceals these relationships. The potential problems of this situation is strikingly emphasized in Fig. 1 . There, $R_{2}\left(\tau_{\mathrm{cp}}\right)$ dispersions are plotted for various cases of two-site exchange: in (A), (B), and (C), $R_{2}\left(\tau_{\mathrm{cp}}\right)$ is plotted for three distinct values of $\Delta \omega, p_{a}$, or $k_{\mathrm{ex}}$, respectively, while the other two parameters are held constant and $R_{2}^{0}=15 \mathrm{~s}^{-1}$ is common to all plots. For these cases, it is clear that the magnitude of $R_{2}\left(\tau_{\mathrm{cp}}\right)$ is primarily determined by $\Delta \omega$ and $p_{a}$, whereas the kinetics $\left(k_{\text {ex }}\right)$ of exchange have a more subtle effect on the NMR data, and the three plots in Fig. $1 \mathrm{C}$ would be indistinguishable at even a modest level of experimental noise. Therefore, confident differentiation between significant changes in exchange rates requires extremely high-quality experimental data or, as we shall see, experiments in addition to the single-field conditions assumed in Fig. 1. 

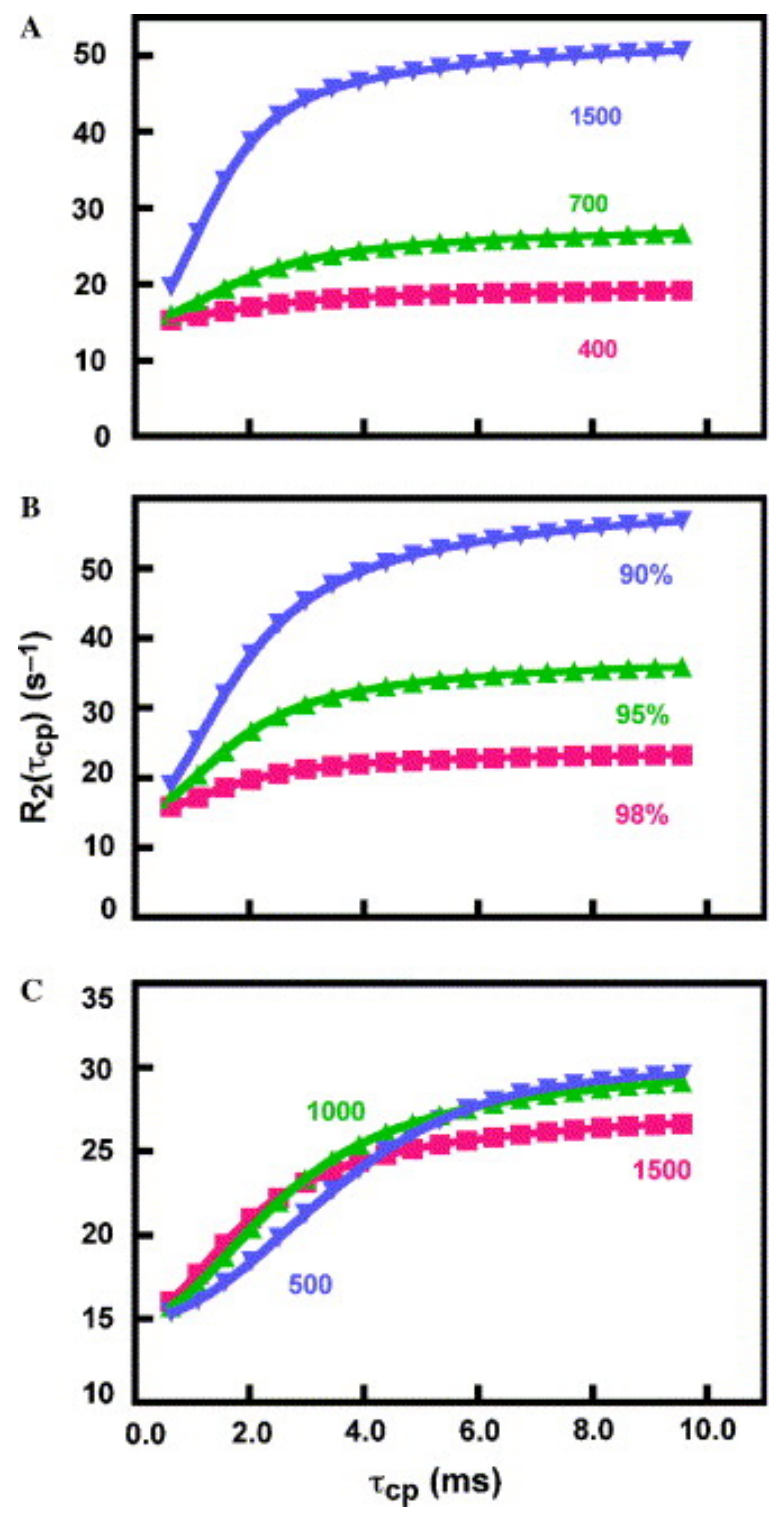

Fig. 1. Parameter dependence of relaxation dispersion curves. In (A) the dependence of $R_{2}\left(\tau_{\mathrm{cp}}\right)$ on $\Delta \omega$, where $\Delta \omega=400 \mathrm{~s}^{-1}$ (red), $700 \mathrm{~s}^{-1}$ (green), and $1500 \mathrm{~s}^{-1}$ (blue), and $k_{\mathrm{ex}}=1500 \mathrm{~s}^{-1}, p_{a}=0.95$, and $R_{2}^{0}=15.0 \mathrm{~s}^{-1}$. $\ln (\mathrm{B})$, the influence of fractional conformer population, $p_{a}=0.90$ (blue), 0.95 (green), and 0.98 (red), where $k_{\mathrm{ex}}=1500 \mathrm{~s}^{-1}, R_{2}^{0}=15.0 \mathrm{~s}^{-1}$, and $\Delta \omega=1000 \mathrm{~s}^{-1}$. In (C), the effect of $k_{\mathrm{ex}}=500 \mathrm{~s}^{-1}$ (blue), $1000 \mathrm{~s}^{-1}$ (green), and $1500 \mathrm{~s}^{-1}$ (red), where $\Delta \omega=700 \mathrm{~s}^{-1}, p_{a}=0.95$, and $R_{2}^{0}=15.0 \mathrm{~s}^{-1}$. (For interpretation of the references to color in this figure legend, the reader is referred to the web version of this paper.)

Closer examination of Fig. 1 provides insight on experimental requirements for accurate exchange determination. For example, to discern between slow and intermediate exchange regimes [blue and green curves in Fig. 1C] it is necessary to have very accurate rate measurements in the fast-pulsing (small- $\tau_{\mathrm{cp}}$ ) region. However, to differentiate intermediate and fast exchange, enhanced quality data is especially important in the slow-pulsing (large- $\tau_{\mathrm{cp}}$ ) region [25]. This can be a conflict (or require extremely high quality across all $\tau_{\mathrm{cp}}$ values) when more than one exchange regime is represented at different residues in the protein. That condition may exist even when the multiple sites are in global exchange (e.g., if $k_{\mathrm{ex}}$ is the same for several residues with distinct $\Delta \omega)$. Furthermore, even barring a variety of exchange types, foreknowledge of the exchange process is not generally possible. Thus, tailored experimental accuracy, say by judicious signal averaging at certain $\tau_{\mathrm{cp}}$ values, is not a generally viable route to faithful parameter estimation. Finally, the assumption in Fig. 1 of matching 
exchange-free relaxation rates, $R_{2}^{0}$, resulted in identical vertical offsets to the curves, but variation in these offsets would not ease distinction of the corresponding exchange parameters, which determine only the shape, not magnitude, of the dispersion. In conclusion, it is important to understand the origins of the similarity in curves such as those in Fig. $1 \mathrm{C}$ for guidance in choosing experiments that present possible solutions to the problem of accurate, faithful parameter estimation.

We now consider the implications of simulation and analysis for the eight combinations of exchange processes and experimental conditions described in Section 2 . The most common method for obtaining estimates of exchange parameters from experimental data is via some type of fitting algorithm. Therefore we simulated this scenario by using synthetic data sets, which additionally allow in-depth analysis of the effects of initial conditions. For each of these cases, we generated 1000 error-incorporated synthetic data sets and used nonlinear least-squares fitting with Eqs. (2) or (8) for two- or three-site exchange, respectively. In addition to this histogramatic analysis of least-squares parameter results, each combination of exchange and experimental conditions was investigated using the direct parameter-mapping approach to reveal parameter correlations and to demonstrate cases in which sufficient experiments were used to remove these correlations and thus successfully estimate the exchange parameters.

\subsection{Two-site exchange with single-field dispersion data}

Single-field simulation results for slow-exchange conditions $\left(k_{\mathrm{ex}}=1000 \mathrm{~s}^{-1}, \Delta \omega=1500 \mathrm{~s}^{-1}, p_{a}=0.95, R_{2}^{0}=15 \mathrm{~s}^{-1}\right)$ are shown in Fig. 2. There, histograms of the parameters that resulted from the 1000 fits clearly indicate that a satisfactory level of accuracy was not possible. The mean (minimum, maximum) of the fitted values are $k_{\mathrm{ex}}=1085 \mathrm{~s}^{-1}\left(144 \mathrm{~s}^{-1}, 2753 \mathrm{~s}^{-1}\right), \Delta \omega=1417 \mathrm{~s}^{-1}\left(654 \mathrm{~s}^{-1}, 2124 \mathrm{~s}^{-1}\right), p_{a}=0.92(0.80,0.96)$, and $R_{2}^{0}=14.6 \mathrm{~s}^{-1}$ $\left(10.0 \mathrm{~s}^{-1}, 19.5 \mathrm{~s}^{-1}\right)$. Strikingly, the distribution of fitted parameters is bimodal for $k_{\mathrm{ex}}, \Delta \omega$, and $R_{2}^{0}$, while the histogram for $p_{a}$ is skewed to small populations relative to the input value. This poor reproduction of the parameters used to generate the data may be attributed to the correlation between the fit parameters. This is apparent from the pairwise plots of the fit parameters in Figs. $3 \mathrm{~A}-\mathrm{C}$, where interdependence is clear from the patterned distribution of results. All possible parameter correlations are significant, with particularly problematic correlation for $\left(p_{a}, k_{\mathrm{ex}}\right)$ and $\left(p_{a}, \Delta \omega\right)$ pairs. As Figs. $3 \mathrm{~B}$ and $\mathrm{C}$ show the latter correlations allow multiple $k_{\text {ex }}$ and/or multiple $\Delta \omega$ values at single values of $p_{a}$. This is also reflected in the bimodal distributions for $k_{\text {ex }}$ and $\Delta \omega$ shown in Figs. $2 A$ and B. 


\section{A}
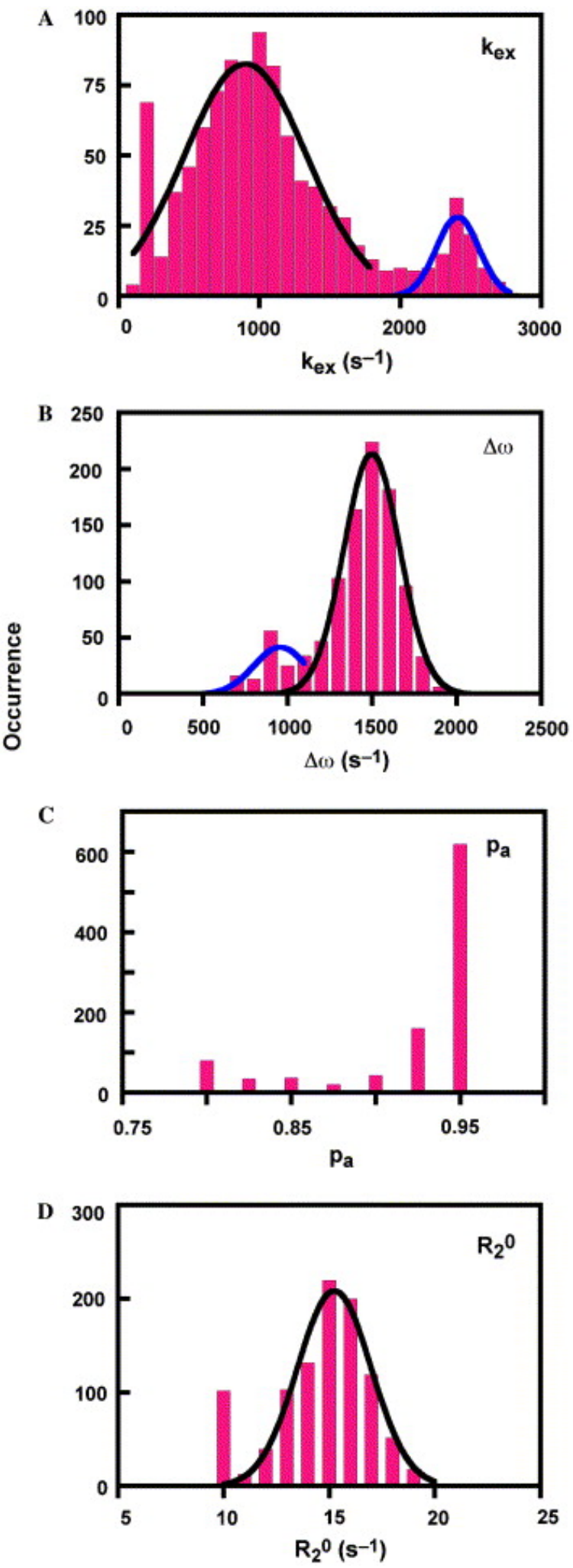

Fig. 2. Parameter distribution (slow exchange). Histograms of parameter estimates from fits of Eq. (2) to 1000 error-incorporated synthetic data sets: (A) kex, (B) $\Delta \omega,(\mathrm{C})$ pa, and (D) $R_{2}^{0}$. Only single-field dispersion was

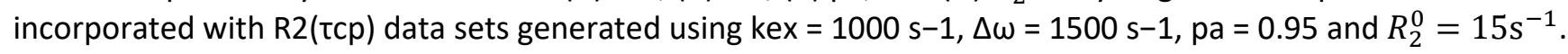
The parameter distributions in (A, B, and D) are modeled with either single or double Gaussian fits. 

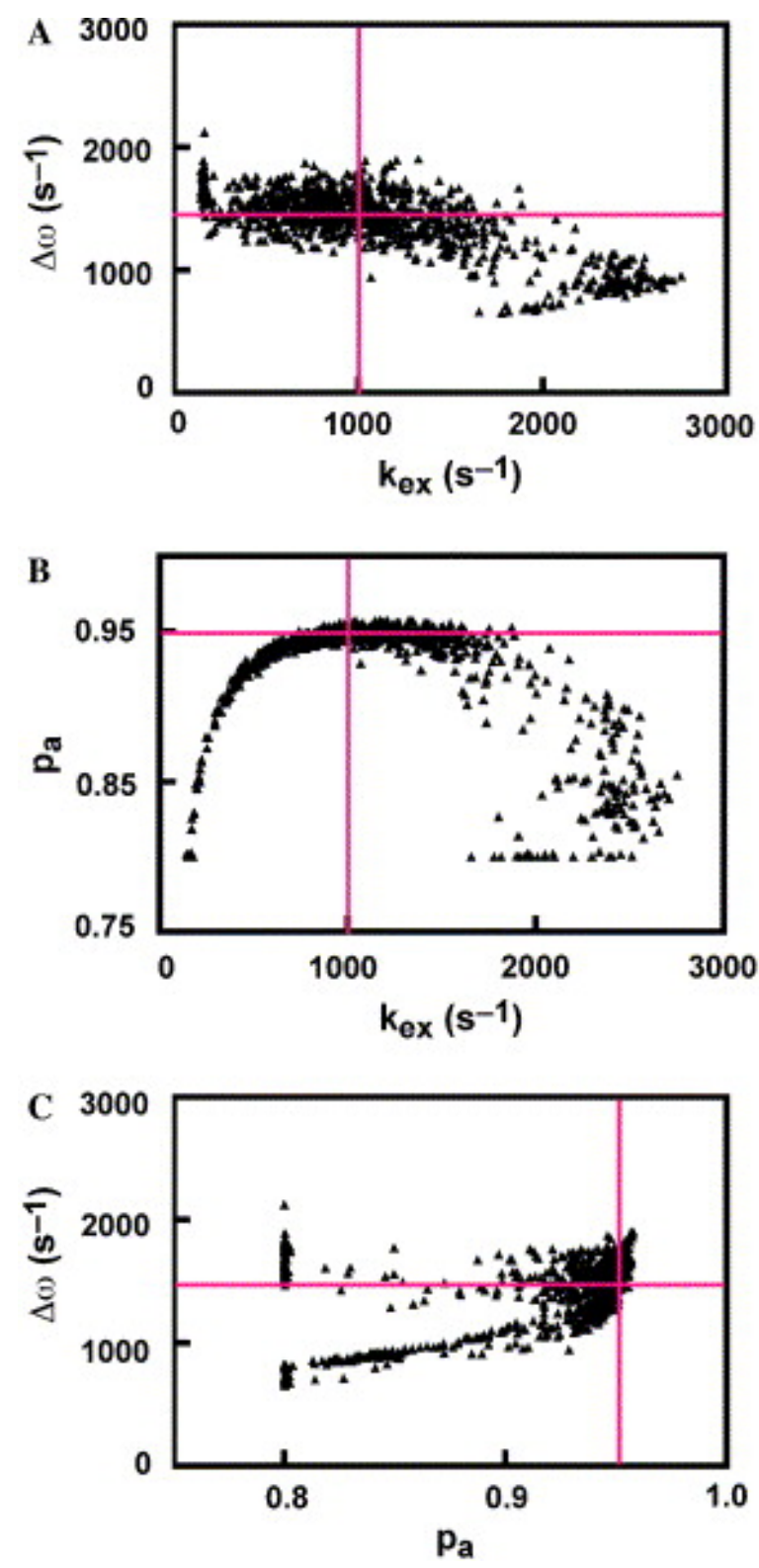

Fig. 3. Parameter correlations. Correlation plots of the same fit results plotted in Fig. 2 . The red lines indicate the unperturbed values of parameters used to generate synthetic data. (For interpretation of the references to color in this figure legend, the reader is referred to the web version of this paper.)

Fig. 2 Fig. 3 suggest there can be serious problems to single-field exchange characterization. Marked emphasis is added to this point by the $R_{2}\left(\tau_{\mathrm{cp}}\right)$ dispersion plots shown in Fig. $4 \mathrm{~A}$. There, three sets of accepted parameters from fits to the synthetic slow-exchange data were used to calculate dispersion with Eq. (2). The dispersions are nearly identical in spite of parameter sets with $k_{\text {ex }}$ ranging more than an order of magnitude $(200,1000$, and $\left.2600 \mathrm{~s}^{-1}\right), \Delta \omega$ varying by a factor of two $\left(1510,1500\right.$, and $\left.790 \mathrm{~s}^{-1}\right)$, and fairly broad variability in $p_{a}(0.83,0.95$, and 0.81 ). Recall, as well, (see Section 2 ) that these $k_{\mathrm{ex}}, \Delta \omega$, and $p_{a}$ combinations are all physically reasonable, and hence prone to unwarranted acceptance and misinterpretation of the observed exchange event. Similar results to those described were obtained for single-field synthetic data from fast or intermediate exchange conditions (not shown). These results make it clear that single-field measurement of $R_{2}\left(\tau_{\mathrm{cp}}\right)$ can yield a significant probability of erroneous parameters with a large difference from physical reality. 

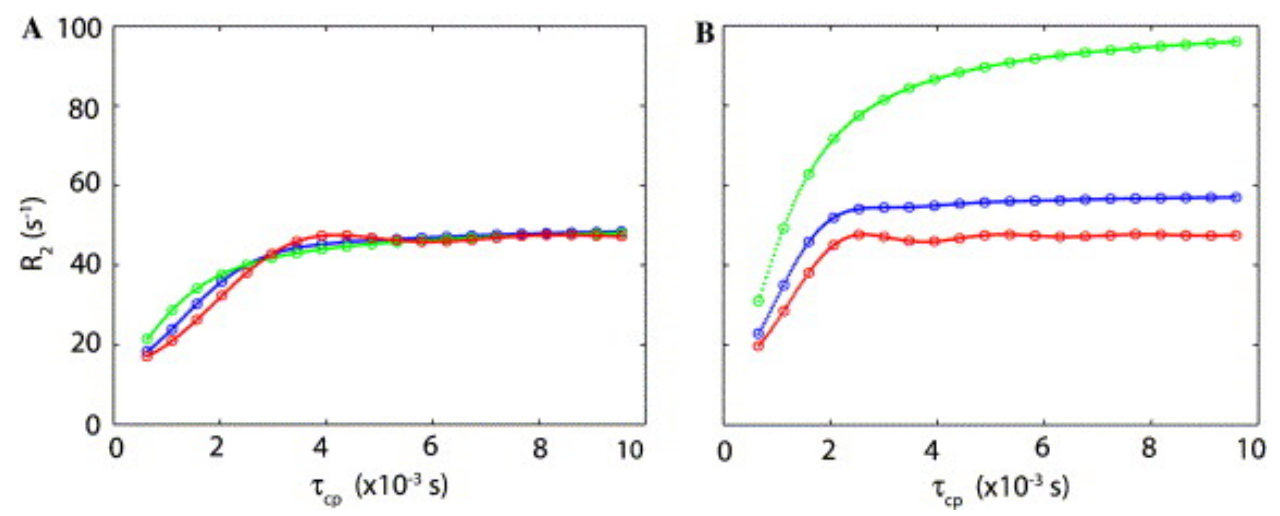

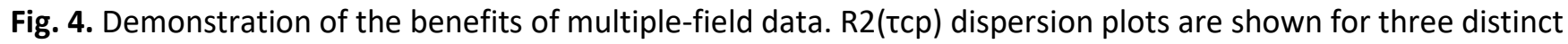
sets of dynamics parameters at both (A) $500 \mathrm{MHz}$ and (B) $800 \mathrm{MHz}$ fields. Parameter sets were chosen to produce similar curves at the higher field. Blue: $k e x=1000 \mathrm{~s}-1, \Delta \omega=1500 \mathrm{~s}-1$, and $p a=0.95$. Green: $k e x=2600$ $\mathrm{s}-1, \Delta \omega=790 \mathrm{~s}-1$, and $\mathrm{pa}=0.81$. Red: $\mathrm{kex}=200 \mathrm{~s}-1, \Delta \omega=1510 \mathrm{~s}-1$, and $\mathrm{pa}=0.834$. In all cases $R_{2}^{0}=15.0 \mathrm{~s}^{-1}$. (For interpretation of the references to color in this figure legend, the reader is referred to the web version of this paper.)

Finally, Fig. 4B demonstrates the great potential of turning from single- to multiple-field dispersion analysis. In contrast to the practically indistinguishable curves from Fig. $4 A$, part $B$ shows the greatly distinct curves calculated with the same exchange parameters as in $\mathrm{A}$, but with data in $\mathrm{B}$ representing $800 \mathrm{MHz}$ data compared to $500 \mathrm{MHz}$ data in $\mathrm{A}$. One might conclude from this that a given set of exchange conditions may be suited to characterization at a particular field. However, the correct field cannot be chosen a priori, nor can it be expected that all exchanging sites in a protein will have the same "ideal" field.

\subsection{Two-site exchange with two-field dispersion data}

Palmer and co-workers [26] have previously noted that multi-field dispersion data enables improved characterization of exchange processes by identifying the timescale of exchange. We have assessed this benefit by generation and analysis of synthetic $R_{2}\left(\tau_{\mathrm{cp}}\right)$ dispersion data at 500 and $800 \mathrm{MHz}$ fields for a single ${ }^{15} \mathrm{~N}$ protein resonance. In this case, the input parameters were $k_{\mathrm{ex}}=1000 \mathrm{~s}^{-1}, p_{a}=0.95$, and $R_{2}^{0}=15.0 \mathrm{~s}^{-1}$, while $\Delta \omega=950 \mathrm{~s}^{-1}$ was used at $500 \mathrm{MHz}$ and scaled by $(800 / 500)=1.6$ at $800 \mathrm{MHz}$. In fitting the synthetic data, we treated $k_{\text {ex }}$ and $\Delta \omega$ and $R_{2}^{0}$ as global (multi-field) fit parameters, imposing the noted linear field dependence on $\Delta \omega$ and incorporating the small field dependence of $R_{2}^{0}$ due to the ${ }^{15} \mathrm{~N}$ CSA.

As shown in Fig. 5 there is a significant improvement in parameter estimation when utilizing data from two fields. The mean ( $95 \%$ confidence interval) values of the fitted parameters for the 1000 data sets are $k_{\mathrm{ex}}=995 \mathrm{~s}^{-1}$ $\left(990-999 \mathrm{~s}^{-1}\right), \Delta \omega(500 \mathrm{MHz})=948 \mathrm{~s}^{-1}\left(947-950 \mathrm{~s}^{-1}\right), p_{a}=0.95(0.9498-0.9499)$, and $R_{2}^{0}(500 \mathrm{MHz})=15.0 \mathrm{~s}^{-1}$ (14.98-15.00 s $\left.\mathrm{s}^{-1}\right)$. Importantly, the fitted parameters are unimodal and well approximated by single Gaussian distributions. Additionally, in the pairwise plots of fitted parameters of Fig. 6 , we see that two-field analysis virtually eliminates non-random parameter correlations, as any small residual correlation is rendered insignificant by its confinement to a very tight parameter subspace. The single-valued character of these correlation plots also confirms the unimodality of the histograms in Fig. 5 . Thus, the parameter ambiguity observed from single-field dispersion data (Fig. 4A), is relieved by two-field acquisition. Finally, extending the analysis to include dispersion data from a third field (not shown) does slightly improve parameter estimation, but the slight gains do not warrant the additional spectrometer time. 
A
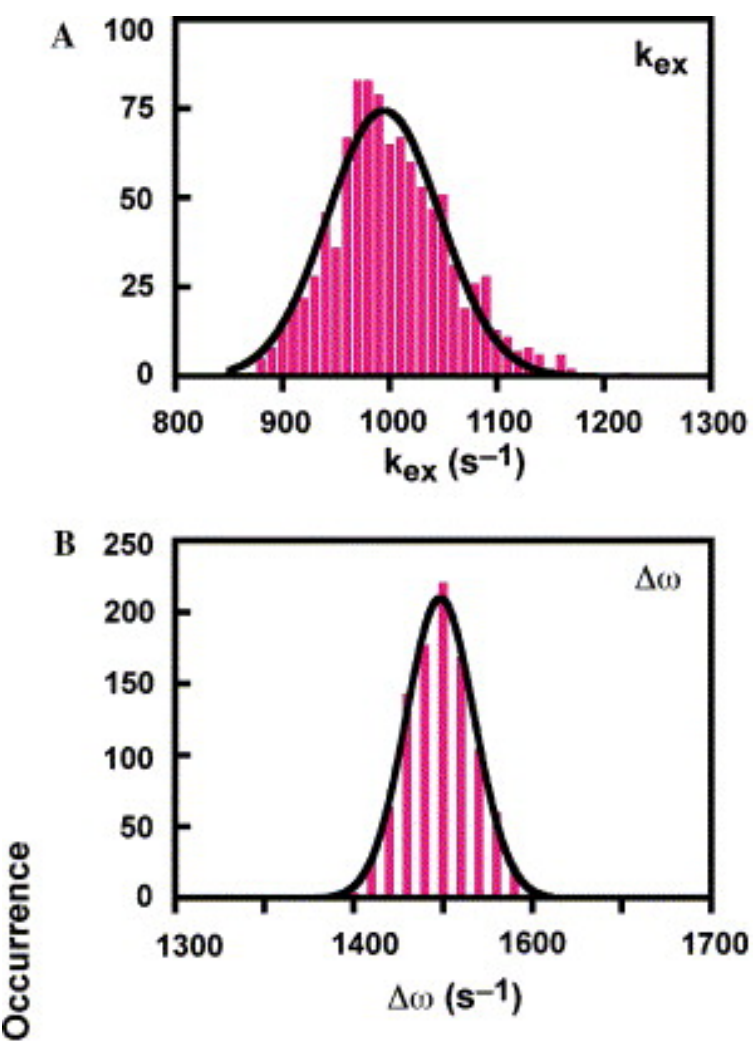

C

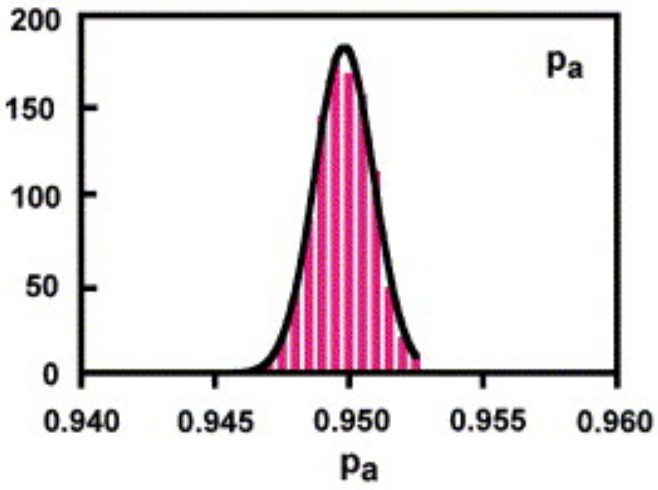

D

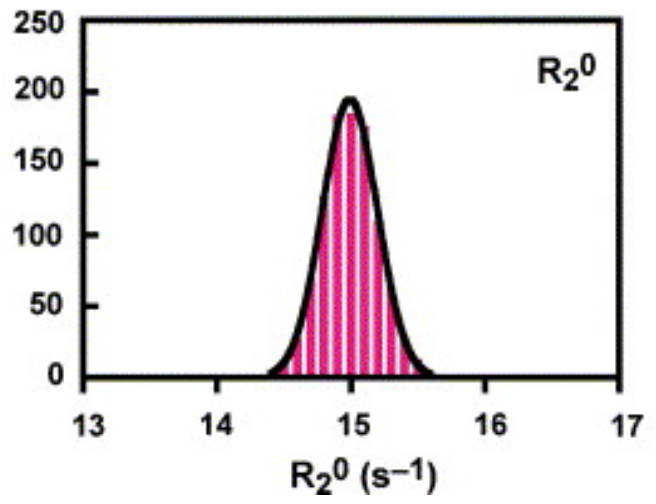

Fig. 5. Parameter distribution (two magnetic fields). Histogram of the parameter estimates for (A) $k_{\text {ex }}$ (B) $\Delta \omega^{18.8 T}$, (C) $p_{a}$, and (D) $R_{2}^{0}$ from fitting Eq. (2) to 1000 synthetic data sets simultaneously at a 11.7 and $18.8 \mathrm{~T}$ magnetic fields. Input parameters were $k_{\mathrm{ex}}=1000 \mathrm{~s}^{-1}, \Delta \omega^{11.7 \mathrm{~T}}=950 \mathrm{~s}^{-1}\left(\Delta \omega^{18.8 \mathrm{~T}}=1500 \mathrm{~s}^{-1}\right), p_{a}=0.95$, and $R_{2}^{0}=15 \mathrm{~s}^{-1}$. The resulting distributions are modeled with a single Gaussians. 

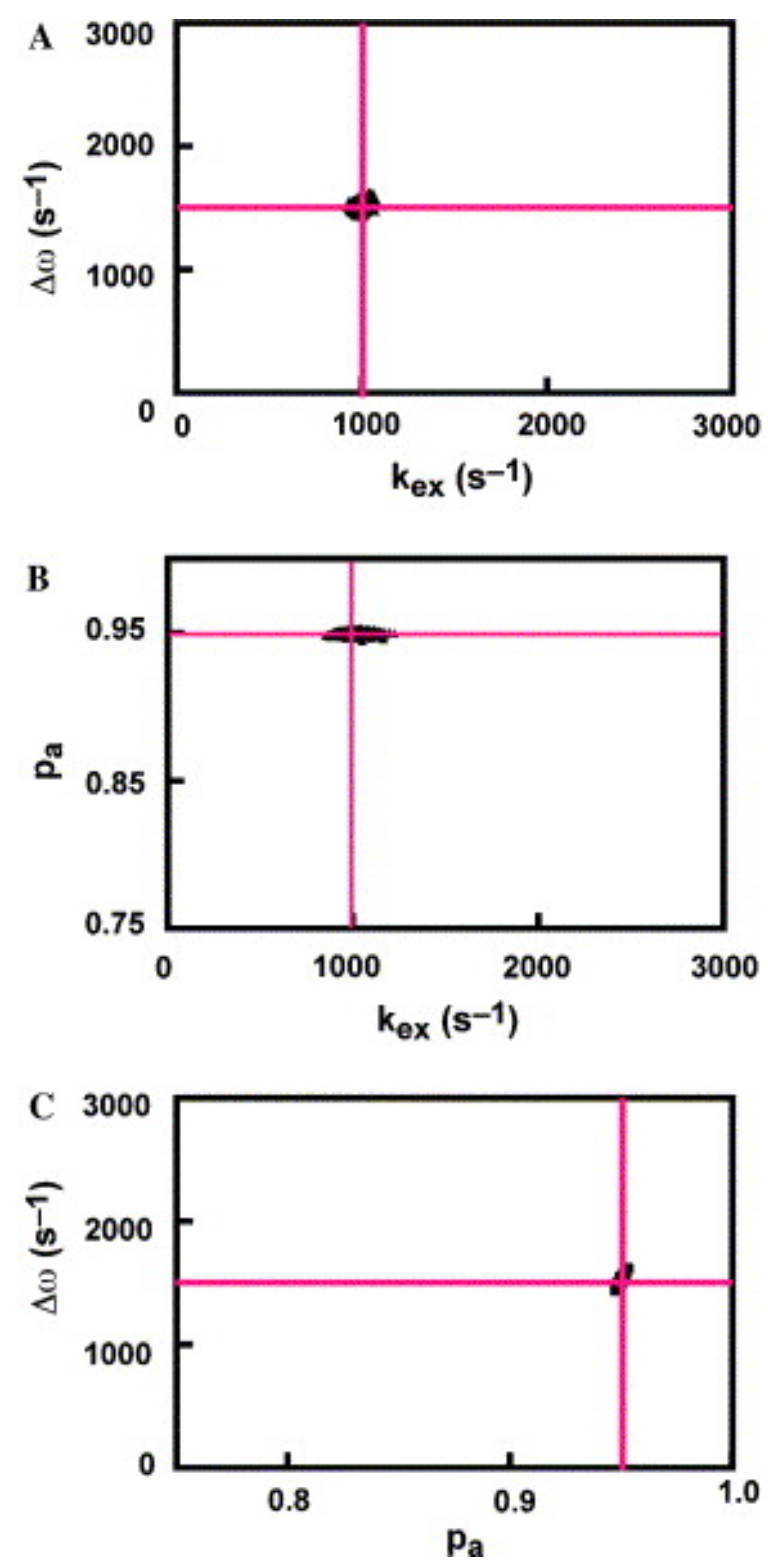

Fig. 6. Parameter correlations. Correlation of the fit parameters from the data in Fig. 5. Pairwise correlations are shown between (A) $k_{\text {ex }}$ and $\Delta \omega$, (B) $k_{\text {ex }}$ and $p_{a}$ and (C) $p_{a}$ and $\Delta \omega$. Red lines indicate the unperturbed values of parameters used to generate synthetic data. (For interpretation of the references to color in this figure legend, the reader is referred to the web version of this paper.)

\subsection{Two-site exchange with single-field dispersion data from three resonances}

In all cases described so far, a single ${ }^{15} \mathrm{~N}$ resonance was considered. Typically for proteins, an exchange event involves several amino-acid residues [1], [6], [27] and often that process is global (i.e., shared $k_{\text {ex }}$ and $p_{a}$ values). In fact, the occurrence of global exchange may enable enhanced parameter estimation, even obviating the need for multi-field data, by expanding the size of the fitted data set relative to the number of fit parameters. Of course, this assumes that multi-residue exchange yields usable NMR data (well-resolved, good S/N and residuespecific values of $\Delta \omega \neq 0$ ) at more than one involved site. Given that, we assessed whether global fits to singlefield dispersion data from three residues can result in sufficiently accurate parameter estimates. In other words, we have asked whether the constraint of shared $k_{\mathrm{ex}}$ and $p_{a}$ with distinct dispersion curves (due to residuespecific $\Delta \omega$ ) leads to accurate reproduction of the synthetic input parameters without resorting to multi-field data. The input parameters used in synthesis of error-incorporated data sets were $k_{\mathrm{ex}}^{\text {global }}=1500 \mathrm{~s}^{-1}, p_{a}^{\text {global }}=$ 
0.95 and $\Delta \omega_{i}=400,600,1500 \mathrm{~s}^{-1}$ and $R_{2, i}^{0}=15,20,25 \mathrm{~s}^{-1}$, respectively, for the three residues (referred to by subscripts $i$ ). Histograms of the 1000 resulting values for each fit parameter are shown in Fig. 7 . For each of $k_{\text {ex }}$ $\Delta \omega_{1}, \Delta \omega_{2}$, and $R_{2,1,2,3}^{0}$, an approximately normal distribution resulted with respective means ( $95 \%$ confidence intervals) of $k_{\mathrm{ex}}=1411 \mathrm{~s}^{-1}\left(1379-1443 \mathrm{~s}^{-1}\right), \Delta \omega_{1}=362 \mathrm{~s}^{-1}\left(362-363 \mathrm{~s}^{-1}\right), \Delta \omega_{2}=546 \mathrm{~s}^{-1}\left(542-550 \mathrm{~s}^{-1}\right), R_{2,1}^{0}=$ $15.10 \mathrm{~s}^{-1}\left(15.0-15.1 \mathrm{~s}^{-1}\right), R_{2,2}^{0}=20.15 \mathrm{~s}^{-1}\left(20.1-20.2 \mathrm{~s}^{-1}\right)$, and $R_{2,3}^{0}=25.20 \mathrm{~s}^{-1}(25.0-25.3 \mathrm{~s}-1)$. Most significant in these results is that the means of kex, $\Delta \omega 1$, and $\Delta \omega 2$ differ by $-6.0,-9.5$, and $-9.5 \%$ from their true values, which are not even encompassed by the corresponding $95 \%$ confidence intervals. Exacerbating this situation, we see in Fig. 7D that the distribution of $\Delta \omega 3$ is bimodal with mean values of 1527 and $895 \mathrm{~s}-1$. Meanwhile, the pa distribution is skewed to low populations, as in the single-resonance, single-field analysis, with $20 \%$ of results significantly different from the test parameters. Finally, the $R_{2, i}^{0}$ means are identical to the inputs. Based on the noted discrepancies between true and fitted $k_{\mathrm{ex}}, \Delta \omega_{1}, \Delta \omega_{2}$, and $\Delta \omega_{3}$ values, we conclude that the additional information available when one assumes global multiple-residue exchange is not necessarily sufficient to neglect the use of multiple fields. We additionally assessed the global exchange scenario by considering three residues, all in the slow exchange limit. Similar results to those described above were obtained and are presented as Supplemental material. Even here, confident estimation of parameters benefits significantly from multi-field experimental data.

A
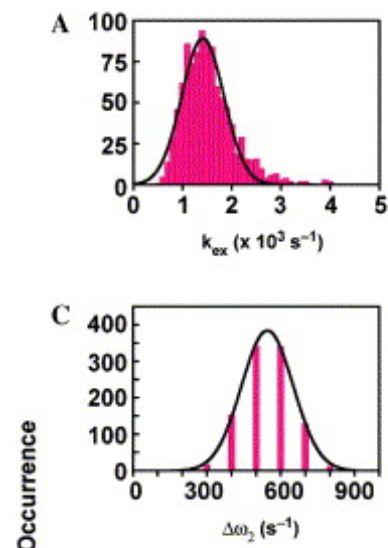

E
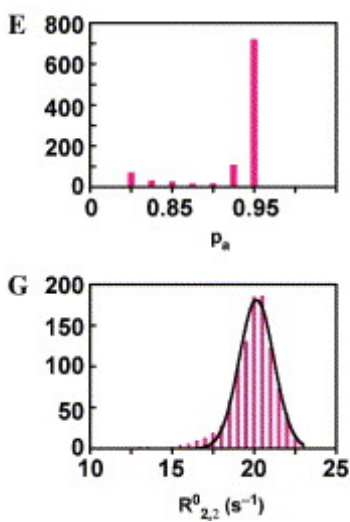
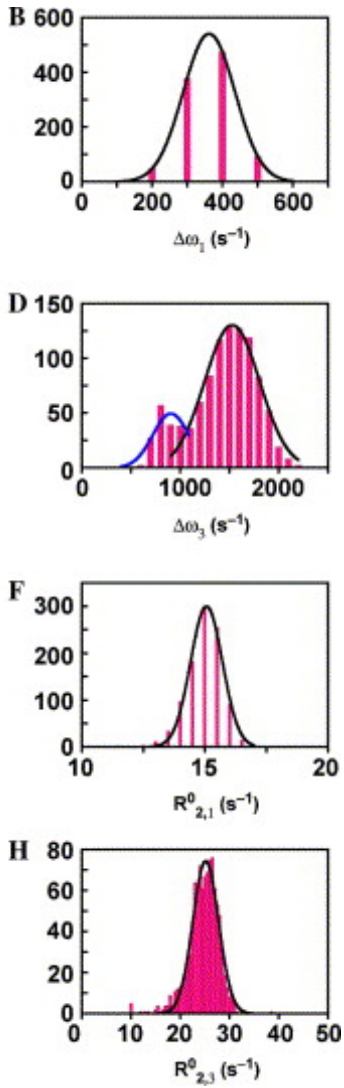

Fig. 7. Parameter distribution (one magnetic field). Histogram of parameter estimates resulting from fits of twosite three-residue global exchange synthetic data to Eq. (2). Results are displayed for (A) $k_{\mathrm{ex}}$, (B) $\Delta \omega_{1}$, (C) $\Delta \omega_{2}$, (D) $\Delta \omega_{3},(\mathrm{E}) p_{a},(\mathrm{~F}) R_{2,1}^{0},(\mathrm{G}) R_{2,2}^{0}$, and (H) $R_{2,3}^{0}$ and the unperturbed inputs were $k_{\mathrm{ex}}=1000 \mathrm{~s}^{-1}, \Delta \omega_{1}=400 \mathrm{~s}^{-1}$, $\Delta \omega_{3}=600 \mathrm{~s}^{-1}, \Delta \omega_{3}=1500 \mathrm{~s}^{-1}, \mathrm{p}_{a}=0.95, R_{2,1}^{0}=15 \mathrm{~s}^{-1}, R_{2,2}^{0}=20 \mathrm{~s}^{-1}$, and $R_{2,3}^{0}=25 \mathrm{~s}^{-1}$.

It is worth noting that, in the case just considered, one of the three residues is near the intermediate exchange regime $\left(k_{\mathrm{ex}}^{\text {global }}=\Delta \omega_{3}=1500 \mathrm{~s}^{-1}\right.$, which may be particularly hard to quantify due to potential poor quality of the NMR lineshape [14]. In addition and as discussed with regard to Fig. 2B, intermediate-exchange dispersion 
overlaps with aspects of both slow- and fast-exchange curves, and may add little to the analysis, suggesting that results from sites apparently in intermediate exchange could be discarded in analysis. A problem with this notion is its assumption that single-field analysis defines the exchange regime. As demonstrated by this analysis thus far and other work [24], this assumption is not valid.

\subsection{Three-site conformational exchange with two-field dispersion data}

Here, we consider three-site linear $(c \leftrightarrow a \leftrightarrow b)$ exchange in the fast exchange limit, as described in Section 2.1 and where dispersion is determined by the sum of $R_{2}^{0}$ and Eq. (8). Because this increases the number of exchange parameters relative to two-site mechanisms without increasing the size of the data set, single-field data is not expected to adequately define three-site exchange. Therefore, we start by evaluating the sufficiency of a two-field approach. Histogramatic analysis (Fig. 8) of fits to the 1000 two-field error-incorporated synthetic dispersion sets demonstrate unimodal, Gaussian distributions with means very close to the input values: $\kappa_{2}=10,055 \mathrm{~s}^{-1}\left(9914-10,195 \mathrm{~s}^{-1}\right), \kappa_{3}=987 \mathrm{~s}^{-1}\left(978-996 \mathrm{~s}^{-1}\right), \phi_{2}=2428 \mathrm{~s}^{-2}\left(2411-2445 \mathrm{~s}^{-2}\right)$, and $\phi_{3}=196 \mathrm{~s}^{-2}(193-$ $200 \mathrm{~s}^{-2}$ ), where the parenthetical ranges are $95 \%$ confidence intervals. Corresponding differences between these values and the input parameters are very small: $0.4,-2.3,0.1$, and $-3.4 \%$. This demonstrates that all values are reproduced with sufficient accuracy in the case of three-site linear exchange. Simulations of spin-relaxation data acquired at three magnetic field strengths (not shown) did not significantly improve parameter estimation.
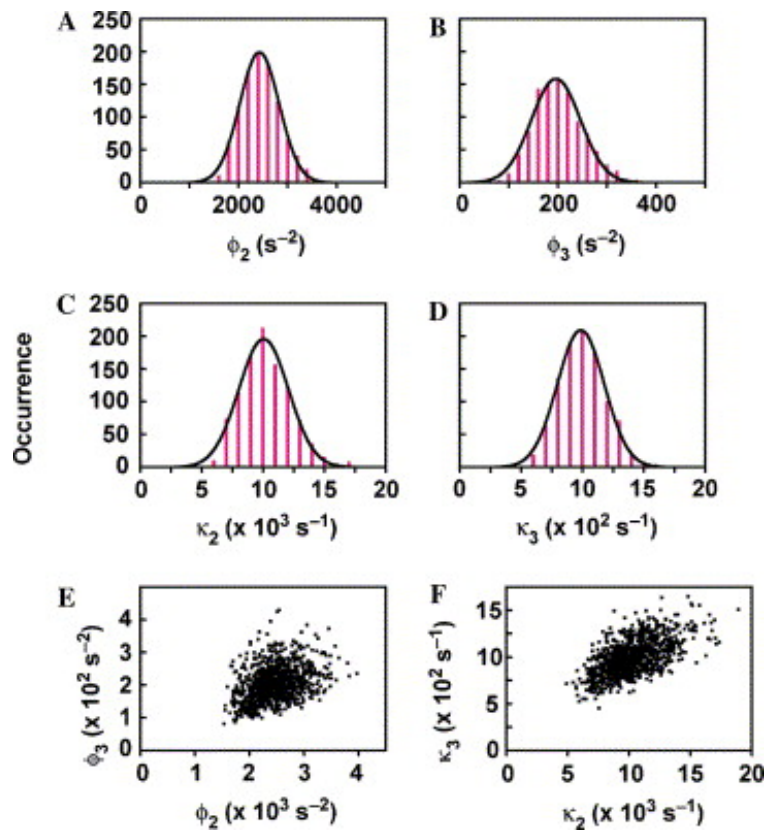

Fig. 8. Three-site fast limit exchange. The results of fitting Eq. (8) to synthetic data sets from two static magnetic fields for a linear three-site exchange process. The input parameters used to generate the data were $\kappa_{2}=10,100 \mathrm{~s}^{-1}, \kappa_{3}=1010 \mathrm{~s}^{-1}, \phi_{2}=2425 \mathrm{~s}^{-2}$, and $\phi_{3}=203 \mathrm{~s}^{-2}$. (A-D) A histogram of the fit parameters for the 1000 data sets for (A) $\phi_{2},(\mathrm{~B}) \phi_{3},(\mathrm{C}) \kappa_{2}$, and (D) $\kappa_{3} . \ln (\mathrm{E})$ and (F) the correlation between $\phi_{2}$ and $\phi_{3}$ and $\kappa_{2}$ and $\kappa_{3}$, respectively, is shown.

\subsection{Direct parameter mapping: characterization of interparameter correlations}

The above analyses address the accuracy of fit results with synthetic data in much the same way that fitting procedures are used to analyze real NMR data. More direct analysis of parameter interdependence in the equations for $R_{2}\left(\tau_{\mathrm{cp}}\right)$ is available using the direct parameter-mapping approach discussed in Section $\underline{2}$. This gridbased approach avoids the bias in initial parameter estimates that is inherent to least squares fitting routines. Thus, while the previous analysis of least-squares results was instructive for its connection to real-data handling, direct parameter mapping provides a comprehensive, unbiased analysis of results. An additional clear advantage 
of direct parameter mapping is seen by inspection of Fig. 9, Fig. 10 as they demonstrate the entire 'equivalence' regions where indistinguishable parameter sets are located. By definition, the space sampling in this approach is uniform and allows constructing accurate maps. The Monte-Carlo fitting approach has no control on where the resulting parameter set is found after the run. Therefore, sampling of the parameter space is highly uneven and must be balanced by an increase in a number of fitting trials that make this approach inefficient for this particular purpose.
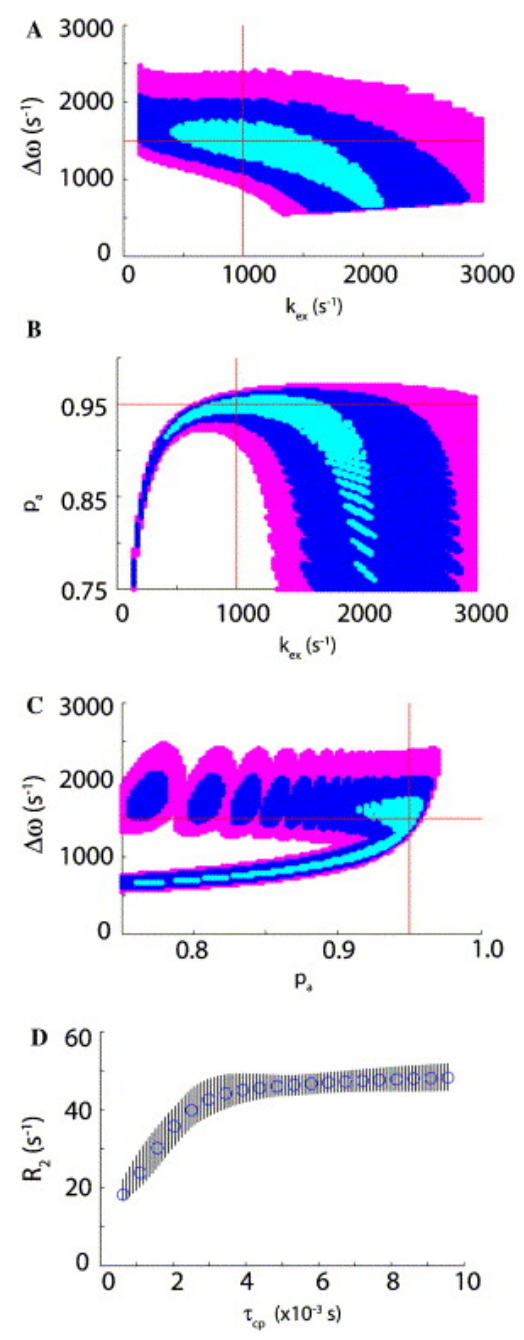

Fig. 9. Parameter mapping for two-site exchange at a single field. Colored regions represent parameter sets found to be indistinguishable from the test set $\left(k_{\mathrm{ex}}=1000 \mathrm{~s}^{-1}, \Delta \omega=1500 \mathrm{~s}^{-1}, p_{a}=0.95, R_{2}^{0}=15 \mathrm{~s}^{-1}\right)$ when the noise RMSDs (percent of $R_{2}$ values) were set to $2.5 \%$ (cyan), $5 \%$ (blue), and $7.5 \%$ (magenta). The regions corresponding to higher uncertainty encompass and include the lower-uncertainty regions. Two-dimensional projections of 3D results are displayed: (A) $k_{\text {ex }}-\Delta \omega$ plane, (B) $k_{\mathrm{ex}}-p_{a}$ plane, and (C) $p_{a}-\Delta \omega$ plane. Input test parameter values are at the intersection of the red lines. In (D), the $R_{2}\left(\tau_{\mathrm{cp}}\right)$ values corresponding to the test parameter set are shown as blue open circles. The shaded area represents the range of $R_{2}\left(\tau_{\mathrm{cp}}\right)$ deviations that result from grid points deemed indistinguishable from the test set at the $5 \%$ level of uncertainty. It should be noted that separate blue areas in (C) are not local minima, but rather a plotting artifact due to narrowing of the $5 \%$ area relative to the grid resolution in $k_{\mathrm{ex}}$. (For interpretation of the references to color in this figure legend, the reader is referred to the web version of this paper.) 

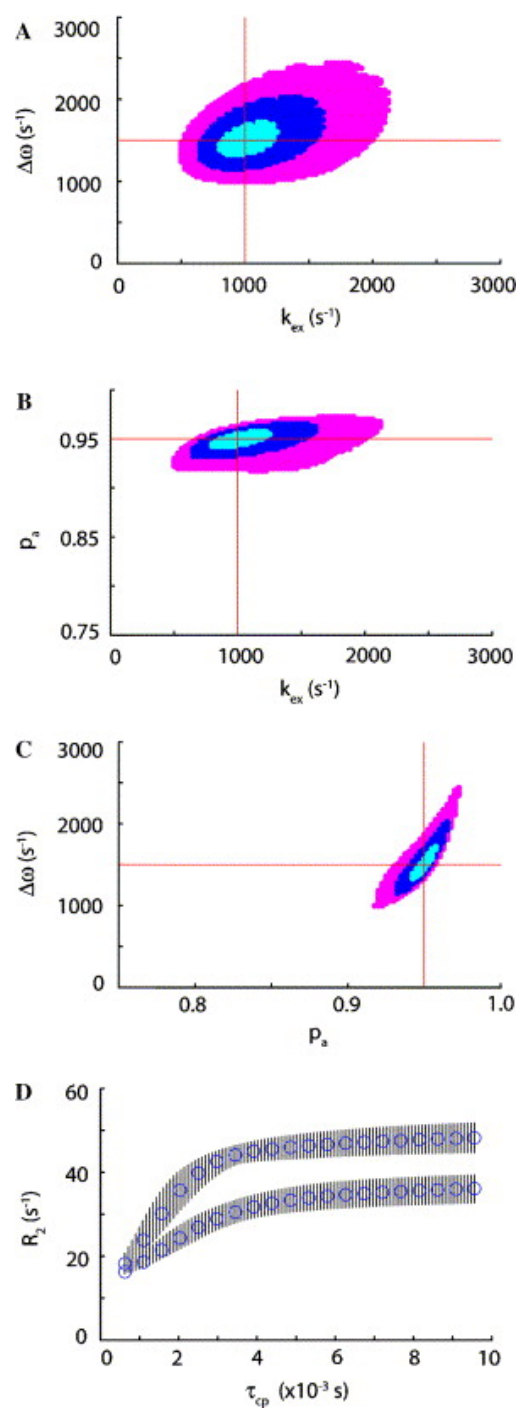

Fig. 10. Parameter mapping for two-site exchange at two fields. Fields used were 500 and $800 \mathrm{MHz}{ }^{1} \mathrm{H}$ frequency and the test set (red lines) was $k_{\mathrm{ex}}=1000 \mathrm{~s}^{-1}, \Delta \omega$ (at $\left.800 \mathrm{MHz}\right)=1500 \mathrm{~s}^{-1}, p_{a}=0.95$, and $R_{2}^{0}=15 \mathrm{~s}^{-1}$ ). As in Fig. 9, color-uncertainty correspondence is $2.5 \%$ (cyan), $5 \%$ (blue), and $7.5 \%$ (magenta) and higher uncertainties encompass and include the lower-uncertainty regions. Two-dimensional projections are shown for (A) $k_{\text {ex }}-\Delta \omega$, (B) $k_{\mathrm{ex}}-p_{a}$ and (C) $p_{a}-\Delta \omega$ planes. In (D), blue open circles are the $R_{2}\left(\tau_{\mathrm{cp}}\right)$ values calculated from Eq. (2) using the test parameters and assuming $800 \mathrm{MHz}$ (upper plot) and $500 \mathrm{MHz}$ (lower plot) fields, while the shaded area shows the range of $R_{2}\left(\tau_{\mathrm{cp}}\right)$ deviations resulting from grid points deemed indistinguishable at the $5 \%$ level of uncertainty. (For interpretation of the references to color in this figure legend, the reader is referred to the web version of this paper.)

The additional information content of the direct parameter mapping results allows one to quantitatively estimate the benefit of using (or not using) the additional field data in every particular exchange mode. The data in Fig. 9, Fig. 10 demonstrate this, where colored 'equivalence' regions are directly compared for different levels of data uncertainty for single and two fields. Such a comparison could provide important information for selection of experimental parameter settings such as number of fields or amount of necessary signal averaging. The goal here is as in the previous sections: to assess those experimental conditions that sufficiently mitigate parameter correlations to enable faithful, accurate exchange characterization.

We use direct parameter mapping primarily to explore correlations between exchange parameters. This is achieved using two-parameter plots of those grid points deemed indistinguishable from the input exchange 
conditions given some level of experimental uncertainty in $R_{2}\left(\tau_{\mathrm{cp}}\right)$ values. These plots are similar to the plots of fit parameters in Fig. 3, Fig. 6, Fig. 8E and F, but, as noted, replacing fit results with unbiased grid-selected parameters provides a more direct characterization of the exchange equations. In the ideal absence of correlation, these two parameter plots should present a normal, two-dimensional Gaussian distribution of points centered on the original synthetic exchange conditions. Any other systematic relationship between parameters indicates that incorrect selection of one parameter can be compensated by an offsetting error in the other. In this way, NMR data can misrepresent the true exchange conditions.

Direct mapping of the 3D parameter space for the two-site exchange (Eq. (2)) with single-field $R_{2}\left(\tau_{\mathrm{cp}}\right)$ data is shown in Fig. 9. These results can be directly compared to those in Fig. 3 . The blue area (including the encompassed cyan region) corresponds to the parameter sets producing $\Delta_{\text {grid }}<\Delta_{5 \%}$. It is reassuring that the similar display of fit results in Fig. 3 indeed closely maps the blue areas in Fig. 9 . Had a sufficiently large sampling of synthetic data sets been used in generating the former set of plots, the character of these two versions of display would be indistinguishable.

Conveniently, we can use direct parameter mapping to predict indistinguishable parameter spaces at various levels of $R_{2}\left(\tau_{\mathrm{cp}}\right)$ experimental uncertainty. This is shown in Figs. 9A-C, where the cyan regions cover grid points indistinguishable from the test parameters when $2.5 \% R_{2}\left(\tau_{\mathrm{cp}}\right)$ uncertainty was assumed. The blue and magenta regions, each including the lower-noise sections, correspond to 5 and $7.5 \%$ uncertainties. Even at the lowest level of uncertainty, there is significant parameter correlation and a broad range of possible parameter results. Fig. $9 \mathrm{D}$ shows the corresponding variation ( $5 \%$ uncertainty level) in $R_{2}\left(\tau_{\mathrm{cp}}\right)$ values as shaded areas about the noise-free test values (blue open circles). It is somewhat worrisome that apparently slight variations in $R_{2}\left(\tau_{\mathrm{cp}}\right)$ lead to the large uncertainties in exchange parameters shown in parts $A-C$ of the figure. The $5 \%$ noise level is not unusual for $R_{2}$ measurements, but, from this analysis of single-field data, it is clearly sufficient to obscure the connection between the dynamic system and the equations of exchange.

We similarly applied direct parameter mapping to the two-field $(500,800 \mathrm{MHz})$ case of two-site exchange. Figs. $10 \mathrm{~A}-\mathrm{C}$ demonstrate the significant reduction in the degree of parameter interdependence that results from incorporating results from an additional field. This result is directly comparable to Fig. 6 , and again, the latter's distribution of fit results is very similar to the blue + cyan ( $5 \%$ uncertainty) areas of Figs. 10A-C. Fig. 10 provides a more complete view of the small residual correlations present with two-field data than was apparent in Fig. 6.

This analysis demonstrates how direct parameter mapping can be used as indication of the degree of confidence appropriate to a given determination of exchange. The single- and two-field determinations here are directly comparable via Fig. 9 , Fig. 10. For example, the areas of the single-field low-uncertainty (2.5\%) regions in Fig. 9 are close to those of the high-uncertainty (7.5\%) two-field results in Fig. 10. Thus analysis of a single-field data may yield quality similar to results obtained utilizing two-field $R_{2}\left(\tau_{\mathrm{cp}}\right)$ data if the experimental uncertainty is reduced at least 3 times. This, of course, would require a ninefold increase in experiment time if one equates $R_{2}$ uncertainty to that of the signal intensities (valid for optimal two-point determination of the rate [14]) Thus, it is much more effective to merely double experiment time using two-field data sets.

In conclusion, we have analyzed parameter interdependences for the equations describing several examples of exchange processes, and uncovered resulting implications for accurate and faithful dynamics characterization by $R_{2}\left(\tau_{\mathrm{cp}}\right)$ dispersion. Using both Monte-Carlo simulations and grid exploration of the full space of exchange parameters, we demonstrated that the high correlation between parameters prevents accurate dynamics parameter estimation. The strong observed interdependence is readily overcome through acquisition of $R_{2}\left(\tau_{\mathrm{cp}}\right)$ dispersion data at more than one static magnetic field, thereby allowing accurate assessment of conformational exchange properties. Our conclusions indicate that two-field dispersion measurement is an important requirement for a reliable dynamics study by NMR. Finally, we have demonstrated that direct parameter 
mapping and comparison can provide a useful indication of the quality of results obtained in a given determination of exchange.

\section{Acknowledgments}

J.P.L acknowledges support from an NSF-CAREER award (MCB-0236966), and funding from the NIH (R01GM070823) and Alfred P. Sloan Foundation. J.G.K. thanks the NIH for a Kirchstein Postdoctoral Fellowship F32GM66599-03.

\section{Appendix A. Supplementary data}

Figure S1. Two-site, three-residue global exchange (slow-exchange regime). Histogram of parameter estimates resulting from fits of two-site three-residue global exchange synthetic data to Equation (2). Results are displayed for (a) $k_{\mathrm{ex}}$, (b) $\Delta \omega_{1}$, (c) $\Delta \omega_{2}$, and (d) $\Delta \omega_{3}$, (e) $p_{\mathrm{a}}$, and the unperturbed input parameters were $k_{\mathrm{ex}}=1000 \mathrm{~s}^{-1}$, $\Delta \omega_{1}=900 \mathrm{~s}^{-1}$ (Red), $\Delta \omega_{2}=1300 \mathrm{~s}^{-1}$ (blue), $\Delta \omega_{3}=1500 \mathrm{~s}^{-1}$ (black), $p_{a}=0.95, R_{2,1}^{0}=15 \mathrm{~s}^{-1}, R_{2,2}^{0}=17 \mathrm{~s}^{-1}, R_{2,3}^{0}=$ $19 \mathrm{~s}^{-1}$. Pairwise correlations of the fit parameters for these exchange conditions is shown between in (f), $k_{\mathrm{ex}}$ and $\Delta \omega,(\mathrm{g}) k_{\mathrm{ex}}$ and $p_{\mathrm{a}}$ and $(\mathrm{h}) p_{\mathrm{a}}$ and $\Delta \omega$. The color scheme for the residue-specific $\Delta \omega$ values is the same as in (b, $\mathrm{c}$, and d).

\section{References}

[1] H. Beach, R. Cole, M. Gill, J.P. Loria Conservation of $\mu s-m s$ enzyme motions in the apo- and substratemimicked state J. Am. Chem. Soc., 127 (2005), pp. 9167-9176

[2] C. Wang, M. Rance, A.G. Palmer Mapping chemical exchange in proteins with MW $>\mathbf{5 0} \mathbf{~ k D ~ J . ~ A m . ~ C h e m . ~ S o c . , ~}$ 125 (2003), pp. 8968-8969

[3] E.Z. Eisenmesser, D.A. Bosco, M. Akke, D. Kern Enzyme dynamics during catalysis Science, 295 (2002), pp. 1520-1523

[4] R. Cole, J.P. Loria Evidence for flexibility in the function of ribonuclease A Biochemistry, 41 (2002), pp. 60726081

[5] L. Wang, Y. Pang, T. Holder, J.R. Brender, A.V. Kurochkin, E.R. Zuiderweg Functional dynamics in the active site of the ribonuclease binase Proc. Natl. Acad. Sci. USA, 98 (2001), pp. 7684-7689

[6] M.J. Osborne, J. Schnell, S.J. Benkovic, H.J. Dyson, P.E. Wright Backbone dynamics in dihydrofolate reductase complexes: role of loop flexibility in the catalytic mechanism Biochemistry, 40 (2001), pp. 9846-9859

[7] R. Ishima, P.T. Wingfield, S.J. Stahl, J.D. Kaufman, D.A. Torchia Using amide $1 \mathbf{H}$ and $15 \mathrm{~N}$ transverse relaxation to detect millisecond time-scale motions in predeuterated proteins: application to HIV-1 protease J. Am. Chem. Soc., 120 (1998), pp. 10534-10542

[8] M. Tollinger, N.R. Skrynnikov, F.A. Mulder, J.D. Forman-Kay, L.E. Kay Slow dynamics in folded and unfolded states of an SH3 domain J. Am. Chem. Soc., 123 (2001), pp. 11341-11352

[9] R.B. Hill, C. Bracken, W.F. DeGrado, A.G. Palmer Molecular motions and protein folding: characterization of the backbone dynamics and folding equilibrium of alpha D-2 using C-13 NMR spin relaxation J. Am. Chem. Soc., 122 (2000), pp. 11610-11619

[10] D.M. Korzhnev, X. Salvatella, M. Vendruscolo, A.A. Di Nardo, A.R. Davidson, C.M. Dobson, L.E. Kay Lowpopulated folding intermediates of Fyn SH3 characterized by relaxation dispersion NMR Nature, 430 (2004), pp. 586-590

[11] S. Kim, C. Bracken, J. Baum Characterization of millisecond time-scale dynamics in the molten globule state of alpha-lactalbumin by NMR J. Mol. Biol., 294 (1999), pp. 551-560

[12] M. Akke NMR methods for characterizing microsecond to millisecond dynamics in recognition and catalysis Curr. Opin. Struct. Biol., 12 (2002), pp. 642-647

[13] T. Mittag, B. Schaffhausen, U.L. Gunther Direct observation of protein-ligand interaction kinetics Biochemistry, 42 (2003), pp. 11128-11136 
[14] J.G. Kempf, J.P. Loria A.K. Downing (Ed.), Protein NMR Techniques, Humana Press, Totowa (2004), pp. 185231

[15] A.G. Palmer, M.J. Grey, C. Wang Solution NMR spin relaxation methods for characterizing chemical exchange in high-molecular-weight systems Methods Enzymol., 394 (2005), pp. 430-465

[16] D.E. Woessner Nuclear transfer effects in nuclear magnetic resonance pulse experiments J. Chem. Phys., 35 (1961), pp. 41-48

[17] L.W. Reeves, E.J. Wells Some steady state and spin echo NMR studies at varied temperatures Disc. Faraday Soc., 34 (1962), pp. 177-184

[18] Z. Luz, S. Meiboom Nuclear magnetic resonance study of the protolysis of trimethylammonium ion in aqueous solution-order of the reaction with respect to solvent J. Chem. Phys., 39 (1963), pp. 366-370

[19] A. Allerhand, H.S. Gutowsky, J. Jonas, R.A. Meinzer Nuclear magnetic resonance methods for determining chemical-exchange rates J. Am. Chem. Soc., 88 (1966), pp. 3185-3193

[20] A. Allerhand, H.S. Gutowsky Spin-echo studies of chemical exchange. II. Closed formulas for two sites J. Chem. Phys., 42 (1965), pp. 1587-1599

[21] J.P. Carver, R.E. Richards A general two-site solution for the chemical exchange produced dependence of $T_{2}$ upon the Carr-Purcell pulse separation J. Magn. Reson., 6 (1972), pp. 89-105

[22] D.G. Davis, M.E. Perlman, R.E. London Direct measurements of the dissociation-rate constant for inhibitor-enzyme complexes via the $T_{1 \rho}$ and $T_{2}$ (CPMG) methods J. Magn. Reson. Ser. B, 104 (1994), pp. 266-275

[23] J. Jen Chemical exchange and NMR T2 relaxation-the multisite case J. Magn. Reson., 30 (1978), pp. 111128

[24] M.J. Grey, C. Wang, A.G. Palmer 3rd.Disulfide bond isomerization in basic pancreatic trypsin inhibitor: multisite chemical exchange quantified by CPMG relaxation dispersion and chemical shift modeling J. Am. Chem. Soc., 125 (2003), pp. 14324-14335

[25] R. Ishima, D.A. Torchia Estimating the time scale of chemical exchange of proteins from measurements of transverse relaxation rates in solution J. Biomol. NMR, 14 (1999), pp. 369-372

[26] O.M. Millet, J.P. Loria, C.D. Kroenke, M. Pons, A.G. Palmer The static magnetic field dependence of chemical exchange linebroadening defines the NMR chemical shift time scale J. Am. Chem. Soc., 122 (2000), pp. 2867-2877

[27] F.A. Mulder, A. Mittermaier, B. Hon, F.W. Dahlquist, L.E. Kay Studying excited states of proteins by NMR spectroscopy Nat. Struct. Biol., 8 (2001), pp. 932-935 